\section{Keine Garantie für Nachhaltigkeit}

\author{
Digitaler Strukturwandel steht teils im Konflikt mit mehr Nachhaltigkeit, teils \\ kann er positiv dazu beitragen. Welcher Effekt überwiegt, hängt ganz wesent- \\ lich von den Anwendungsbedingungen ab. Dies wird anhand des Strukturwan- \\ dels in der Produktion und eines sich stark verändernden traditionellen Sek- \\ tors, der Druckindustrie, erläutert.
}

\section{W}

Von Simone Kimpeler und Frieder Meyer-Krabmer renden Märkten. Dieses führt aber keineswegs zu einer Verdrängung der Produktion. Auch die Erstellung von Dienstleistungen ist nicht ohne den Einsatz physischer Güter möglich. Es kommt daher eher zu Veränderungen der Produktionsprozesse und Absatzstrategien als zu einem Ersatz der physischen Produkte.

Der verstärkte Einsatz von Informationstechnologien (IT) in alltäglichen Geschäfts- und Produktionsprozessen hat eine größere Unabhängigkeit von Ort und Zeit und eine Ausweitung des Wirtschaftsraumes zur Folge. Mehr und mehr Unternehmen arbeiten in virtuellen Unternehmensverbünden mit lokalen Ressourcen für globale Märkte. Ihre Wettbewerbsfähigkeit hängt nicht nur von Skalenerträgen, sondern zusätzlich von Netzwerkeffekten ab. Auf den Absatzmärkten mindern individualisierbare Angebote (Stichwort: Mass Customization) und die Digitalisierung und Vernetzung der Kundendaten die Bedeutung von Intermediären. Der Hersteller kann einen direkten Kundenkontakt pflegen, was zwar dem Produkt zugute kommt, aber den klassischen Einzelhandel außen vor lässt. sensbasienten Diensteistungen und konvergie-
In der New Economy wird also die Wirtschaft nicht neu erfunden, sondern ein digitaler Strukturwandel eingeleitet, der sich ausgehend von der IT-Wirtschaft nach und nach in alle Branchen ausbreitet. IT wird dabei nicht nur innerhalb der Unternehmen sondern auch extern, zwischen den Unternehmen, zur Verbesserung des Informationsflusses und der Produktionsprozesse eingesetzt. Es werden administrative Prozesse automatisiert, die Supply Chain rekonfiguriert, die Steuerung und Automatisierung von Produktionsanlagen optimiert und der Beschaffungswettbewerb intensiviert (siehe Abb. 1). Im Bereich des Personalmanagements wird IT zur Qualifizierung und Weiterbildung sowie für das Wissensmanagement eingesetzt.

Die Innovationen im Bereich der IT und der Softwareentwicklung haben nicht nur die IT-Wirtschaft gestärkt, sondern ermöglichen auch in anderen Branchen die Erschließung neuer Geschäftsfelder. Schon heute besteht die Softwareindustrie zu 45 Prozent aus Unternehmen der so genannten Sekundärbranchen der Softwareindustrie, wie Maschinenbau, Elektronik, Fahrzeugbau, Telekommunikation und Finanzdienstleistungen. Diese Firmen, in der Mehrzahl mittlere bis größere Unternehmen, betreiben die Entwicklung und Anpassung der von ihnen für die

\section{Abbildung 1: Restrukturierung der Prozesse durch den IT-Einsałz}

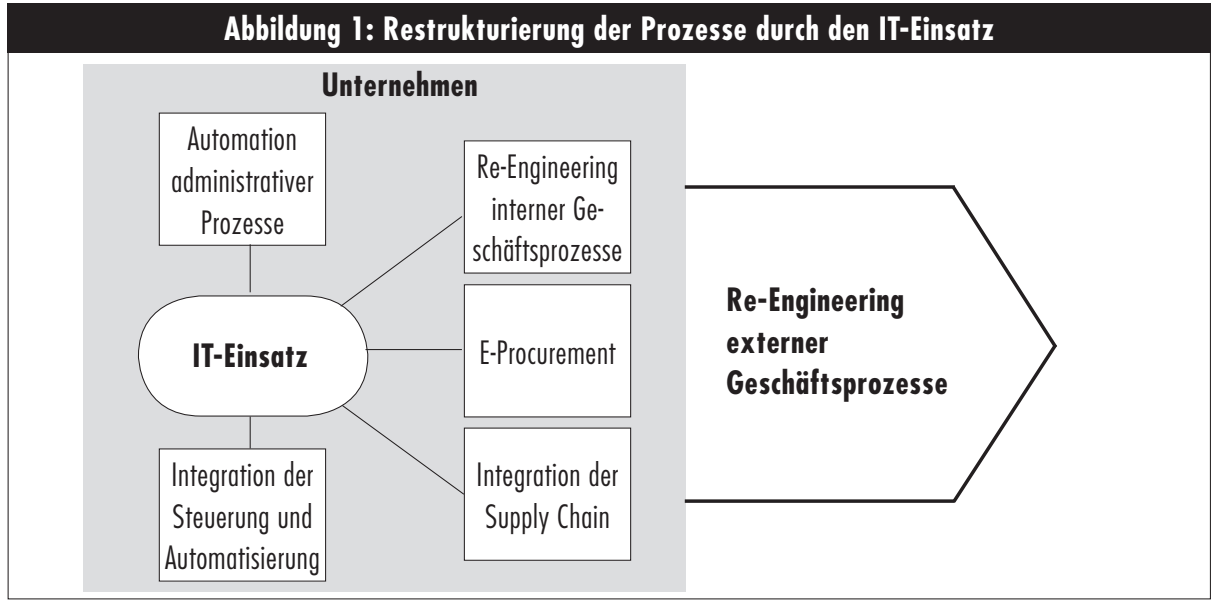

Quelle: eigene Darstellung
Produktion bzw. Ausführung der Dienstleistung benötigten Software selbst (1). Ingenieurmäßige Individuallösungen - zum Beispiel sichere Steuerungsfunktionen wie $\mathrm{ABS}$, umweltschonender Energieverbrauch durch Einspritzsysteme oder Online-Diagnosesysteme - tragen dazu bei, dass diese Branchen ihre Weltmarktführerschaft ausbauen können. Die Entscheidung, die Software im eigenen Haus zu entwickeln, ist abhängig von der wettbewerbssichernden Funktion der Software und ihrem Bezug zur Kernkompetenz des Unternehmens.

Zwischenfazit: Der digitale Strukturwandel löst einen enormen Wachstumsschub aus. Dies wird sicherlich in vielen Bereichen unmittelbar in Konflikt mit den Zielen einer nachhaltigen Entwicklung stehen, wie es aus der Vergangenheit anlässlich anderer Wachstumsschübe bereits bekannt ist. Es eröffnen sich aber auch Chancen, wenn neben den technischen auch organisatorische und soziale Innovationen erfolgen, auf die wir im Folgenden exemplarisch eingehen.

\section{Nachhaltige Dienstleistungen in der Produktion}

Durch die Etablierung von Nachhaltigkeitskonzepten können sich für Unternehmen neue Geschäftsfelder in Form von neuen Produktnutzungsformen und geänderten Angebotsstrategien eröffnen (2). Dabei verschiebt sich der Schwerpunkt der unternehmerischen Tätigkeit von der Produktion der Gebrauchsgüter hin zum Produktmanagement, welches neben der Herstellung auch den Betrieb und Vertrieb sowie Wartung und Betreuung umfasst. Dies bedingt eine weitgehende Herstellerhaftung, da Produkte mit hoher $\mathrm{Zu}$ verlässigkeit, Fehlertoleranz und Schutz gegen Missbrauch nachgefragt werden, die zwangsläufig eine erhöhte Langlebigkeit aufweisen. Um dennoch künftigen technologischen Anforderungen gerecht zu werden, müssen die Produkte zu anpassbaren Komponenten werden, die flexibel in neue Systeme integriert werden können, ohne dass ganze Systemerneuerungen notwendig wären. Somit steht nicht die Qualität der Produkte sondern die Qualität der Nutzung im Mittelpunkt der Produkt- und Innovationspolitik nachhaltig agierender Unternehmen (3).

Die Möglichkeiten nachhaltiger Produktpolitik zur Nutzungsintensivierung und Lebensdauerverlängerung durch ergänzende Dienstleistungsangebote können nur Unternehmen mit entsprechender Produktpalette und Kundenstruktur ökonomisch sinnvoll anbieten. Es zeichnet sich 
langsam ein Wandel in der Produktpolitik der Unternehmen ab. Dies lässt jedoch keineswegs den Schluss zu, dass das Konzept der Nachhaltigkeit in der Produktion als etabliert gilt. Bei den Angebotserweiterungen um zusätzliche Dienstleistungen stehen oft die ökonomischen Interessen den ökologischen Wünschen entgegen und sind noch unvereinbar mit bestehenden Beschäftigungsstrukturen. So verlangen neue Dienstleistungskonzepte nach zusätzlichen Qualifikationen der Mitarbeiter und nach einer Flexibilisierung der Arbeitszeit und des Personaleinsatzes.

Fazit: Trotz bestehender wirtschaftlicher, sozialer und politischer Hürden ist von einer strategischen Umorientierung der Produktionsgüterindustrie hin zu Nachhaltigkeit in der Produktion eine insgesamt positive Wirkung auf die gesamtwirtschaftliche Entwicklung zu erwarten. Eine De-Materialisierung durch Digitalisierung zeichnet sich $a b$, die die materielle Produktion zum Teil durch Dienstleistung substituiert, aber dadurch auch tegrierte Produktion haben Automatisierungsansätze den gesamten Druckprozess weiter digitalisiert. Wichtigste Voraussetzung hierfür ist die Standardisierung der Datenformate.

Unternehmen in der Druckindustrie haben sich zu Mediendienstleistern entwickelt, die so genannte Cross-Media-Services anbieten. Dabei wird der Inhalt des bisher als Druckerzeugnis produzierten Angebots digital aufbereitet und kann unterschiedlich dargestellt, verbreitet und rezipiert werden. Aber trotz der Möglichkeit des Ersatzes von Druckprodukten durch elektronische Alternativen in Teilmärkten ist mit einer weitgehenden Substitution in den nächsten Jahren nicht zu rechnen, und auch die Einbußen der Nutzungszeit von Druckerzeugnissen gegenüber digitalen Medien werden sich mittelfristig in Grenzen halten.

Zwar ist eine vollständige Digitalisierung der Produktionskette in der Druckindustrie technologisch bereits möglich, ihre breite Realisierung wird jedoch noch einige Zeit andauern. War es bis jetzt

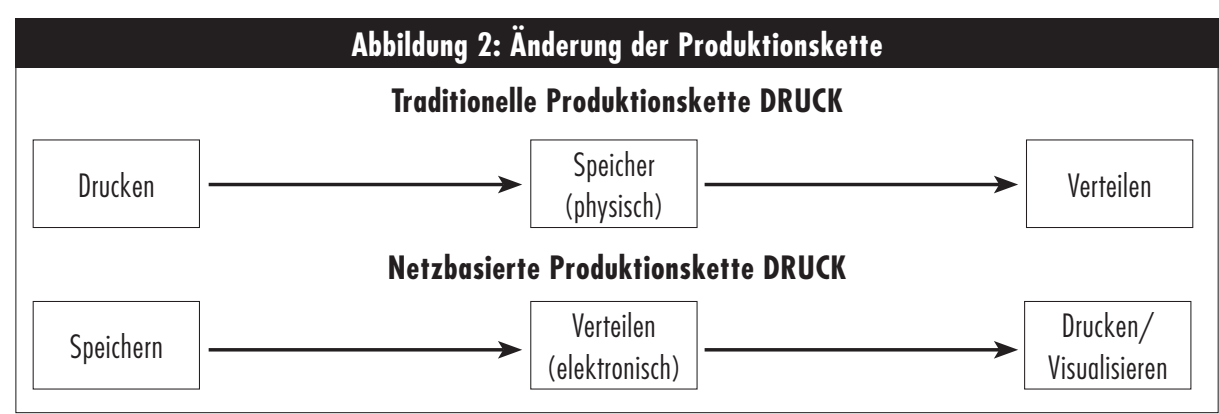

Quelle: eigene Darstellung

beschäftigungsintensiver und stärker inlandsbasiert wird. Entscheidend wird sein, inwieweit sich die geschilderten Veränderungen der Dienstleistungen, der Geschäftsmodelle der Unternehmen, der Nachfrage von Wirtschaft und privaten Haushalten sowie der Arbeitsbedingungen auch tatsächlich durchsetzen.

\section{Das Beispiel Druckindustrie}

Seit Mitte der 80er-Jahre hat IT auch auf die Druckindustrie einen wachsenden Einfluss ausgeübt (4). Waren zuerst nur Vorstufen wie die Datenverarbeitung und Filmbelichtung betroffen, so hat sich der IT-Einsatz sehr bald auch in der eigentlichen Drucktechnik etabliert. Heute sind Mikroprozessoren zur Steuerung von Druckmaschinen nicht mehr wegzudenken. Hinzu kommen die Digitalisierung der Kundendaten und die Einführung entsprechender Grafik-Programme und von Desktop Publishing, sowie elektronische Bildbearbeitungssysteme. Im Bereich Arbeitsabläufe und in- üblich, zuerst zu drucken und dann zu verteilen, so wird durch die digitale Form der Druckvorlagen ein vorausgehendes Verteilen und anschließendes Drucken vor Ort möglich ( Print on Demand, vgl. Abb. 2).

Fazit: Gerade für eine traditionelle Branche wie die Druckindustrie besteht Potenzial, durch den digitalen Strukturwandel die Ressourceneffizienz und somit Nachhaltigkeit zu erhöhen, auch wenn der Digitalisierungsprozess noch nicht abgeschlossen ist. Der Einfluss auf die Wertschöpfungskette ist hier besonders hoch. Es werden kurzfristigere Reaktionen auf die Nachfrage möglich, was sich auf Lagerhaltung und Vertrieb auswirkt. Durch Kooperation mit anderen können die Betriebe ergänzende Dienstleistungen anbieten und somit von der zu beobachtenden Konvergenz im Mediensektor profitieren.

\section{Fazit}

Wir haben versucht, das Doppelgesicht des digitalen Strukturwandels zu verdeutlichen. Einige empirische Schlaglichter haben illustriert, wie er einer nachhaltigen Entwicklung schaden und wie er ihr nützen kann. Welche der zwei Seiten überwiegt, wird von den Bedingungen abhängen, die den digitalen Strukturwandel bestimmen. Diese sind nicht vorrangig technischer Natur, sondern bilden sich im Zusammenspiel von Wirtschaft, Politik und Gesellschaft. Notwendig für einen nachhaltigen digitalen Strukturwandel sind globale und komplexe Systeminnovationen (5). Hierfür sind offensives Lernen durch vielfältige Feldversuche und Pilotvorhaben zum Finden technischer, wirtschaftlicher, rechtlicher und sozialer Lösungen wesentlich.

\section{Anmerkungen}

(1) Friedewald, M. et al.: Softwareentwicklung in Deutschland - Eine Bestandsaufnahme. In: Informatik-Spektrum, Jg. 24 (2001), Heft 4, S. 81-90.

(2) Schirrmeister, E./ Dreher, C./ Fleig, J.: Neue Nutzungsformen, Bericht im Rahmen von "Forschung für die Produktion von morgen", ISI, Karlsruhe 2000.

(3) Meyer-Krahmer, F./ Lay, G.: Der Stellenwert innovativer Dienstleistungen in der Modernisierungsdebatte. In: WSIMitteilungen, Nr. 06/2001, S. 396-400. Vgl. dazu auch den Schwerpunkt Ökoeffiziente Dienstleistungen der Ausgabe $5 / 2000$ von Ökologisches Wirtschaften.

(4) Zu den Auswirkungen des digitalen Strukturwandels auf die Druckindustrie hat das Fraunhofer ISI in den letzten Jahren mehrere Studien durchgeführt, vgl. z.B. Hudetz, W./ Schünke, P./ Georgieff, P.: Druckindustrie im Wandel. Entwicklung und Perspektiven unter dem Einfluss der Informations- und Kommunikationstechnologien. Stuttgart 2000.

(5) Meyer-Krahmer, F./ Kuhlmann, S.: Internationalisation of Innovation, Interdependence, and Innovation Policy for Sustainable Development. In: Sweeney, G. (Hrsg.): Innovation, Economic Progress and the Quality of Life, Cheltenham 2001, S. 86-110.

\section{Die AutorInnen}

Simone Kimpeler ist stellvertretende Leiterin der Abteilung Informations- und Kommunikationssysteme, Prof. Dr. Frieder Meyer-Krahmer ist Leiter des Fraunhofer-Instituts für Systemtechnik und Innovationsforschung (ISI).

Kontakt: ISI, Breslauer Straße 48, 76139 Karlsruhe. Tel. 0721/ 6809-0, Fax -689152, E-mail: ski@isi.fhg.de 
(c) 20I0 Authors; licensee IÖW and oekom verlag. This is an article distributed under the terms of the Creative Commons Attribution Non-Commercial No Derivates License (http://creativecommons.org/licenses/by-nc-nd/3.o/), which permits unrestricted use, distribution, and reproduction in any medium, provided the original work is properly cited. 